\title{
Abteilung Haut und Liebe - Die Geschichte der Hautklinik am Klinikum Nürnberg
}

\author{
Division Skin and Love - History of the Department for Dermatology and Venerology at Nuremberg Hospital
}

Autor

Institut

\section{Debus}

Klinikum Nürnberg

\section{Bibliografie}

DOI http://dx.doi.org/

10.1055/s-0029-1244136

Akt Dermatol 2010; 36:

268-277 @ Georg Thieme

Verlag KG Stuttgart · New York

ISSN 0340-2541

\section{Korrespondenzadresse}

Dr. med. Dirk Debus

Oberarzt der Hautklinik

Klinikum Nürnberg

Prof.-Ernst-Nathan-Straße 1

90419 Nürnberg

dirk.debus@klinikum-

nuernberg.de

\section{Zusammenfassung \\ $\nabla$}

Die Hautklinik am Klinikum Nürnberg blickt im Jahr 2010 auf eine 165-jährige Geschichte zurück. Die Vorgängeranstalt, das sog. „Institut für unheilbar Kranke“, 1813 im Sebastianspital eingerichtet, wurde 1845 durch eine Abteilung für Haut- und Geschlechtskrankheiten im neu errichteten Allgemeinen Städtischen Krankenhaus an

\section{Sebastianspital und „Institut für unheilbar Kranke“}

Der 1806 als Kreismedizinalrat berufene Dr. Wilhelm von Hoven bezeichnete die Zustände der Einrichtungen, die in Nürnberg der Unterbringung von Kranken dienten, als „kaum zu einem Aufenthalt für Verbrecher, vielweniger für Kranke“ geeignet [1]. Erst auf beharrliche Initiative von Hovens wurde 1813 im Heilig-Geist-Spital ein „Krankeninstitut für heilbare Kranke“ eingerichtet und in Ergänzung für diejenigen, die an „ekelhaften Krankheiten“ litten, nämlich für „Krätzige, Syphilitische, Krebsübel, Epilepsie und solche Kranke, die incurabel und auffallend unreinlich waren“ [2], das Sebastianspital im Stadtteil St. Johannis als „Institut für unheilbar Kranke“. Dieses konnte 50 bis 60 Kranke aufnehmen.

Beide Krankenanstalten waren lediglich als Provisorien konzipiert worden bis zur Errichtung eines allgemeinen Krankenhauses. Doch blieben sie über die nächsten 30 Jahre die einzigen Krankenanstalten in Nürnberg. Das Sebastianspital (im Volksmund „Wastl“) für Syphilitische und Hautkranke wurde ab 1836/37 von Dr. Ludwig Bock, zuvor von seinem Vorgänger Dr. Ferg, und von Schwestern des Martha-Maria-Vereins betreut. Wegen ständiger Überfüllung und hoher Mortalität stand das Sebastianspital in der Kritik. Bock hatte nach seinem Dienstantritt die sanitären $\mathrm{Zu}$ stände, die Pflege und vor allem die medizinische der Sandstraße abgelöst. Seit 1897 besteht die Abteilung am Neuen Städtischen Krankenhaus in St. Johannis fort, dem heutigen Klinikum Nürnberg Nord. Nach 110 Jahren im gleichen Gebäude erfolgte 2007 der Umzug in neu renovierte Räume im sog. Y-Bau. Im Folgenden wird die Klinikentwicklung mit Bezug auf einige der medizinischen und gesundheitspolitischen, baulichen und personellen Rahmenbedingungen dargestellt.

Behandlung zwar verbessert, war aber trotzdem der Ansicht, dass die Anstalt weit hinter den aktuellen Anforderungen zurückbleibe, da viele Neuansätze aus Kostengründen nur mangelhaft oder ansatzweise durchgeführt werden könnten [3]. $10-30 \%$ der Behandelten waren syphilitisch, die weiblichen Patienten waren überwiegend Prostituierte, was ,jeder halbwegs noch Ehrgefühl habenden weiblichen Person den Aufenthalt daselbst höchst peinigend machen muss: es trifft sich nemlich gar oft, dass eine so eben aus dem Zuchthaus kommende oder ins Zuchthaus abgeliefert werdende Person neben das unbescholtenste Mädchen zu liegen kommt. Wir müssen aufnehmen, was kommt“ [2]. Bei der Syphilisbehandlung kamen Quecksilber-(Sublimat-)Bäder, Schwefel und Sassaparill zur Anwendung [2].

\section{Das Allgemeine Städtische Krankenhaus an der Sandstraße}

Mit der Errichtung des Allgemeinen Städtischen Krankenhauses im Jahr 1845, nach 17 Jahren Planungs- und Bauzeit, begann in Nürnberg das moderne Krankenhauswesen: „Den 15. Oktober wurde nunmehr das Haus mit 35 Kranken aus den bisherigen Krankenanstalten: dem sogenannten ,Krankeninstitut', einer im Jahre 1813 im Hofspital zum heiligen Geist errichteten Krankenanstalt für medizinische und chirurgische 


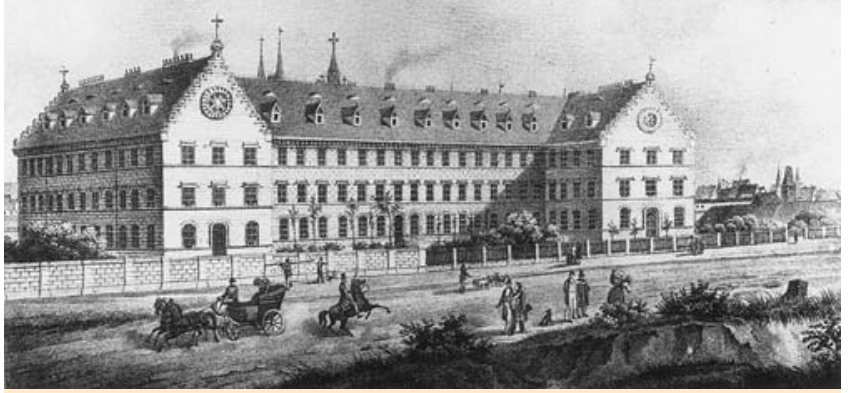

Abb. 1 Das allgemeine Krankenhaus der Stadt Nürnberg an der Sandstraße, Südansicht (aus [5]). Rechts vom linken Giebel erkennt man die beiden Turmspitzen der Lorenzkirche.

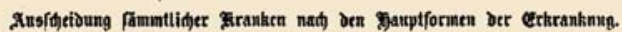

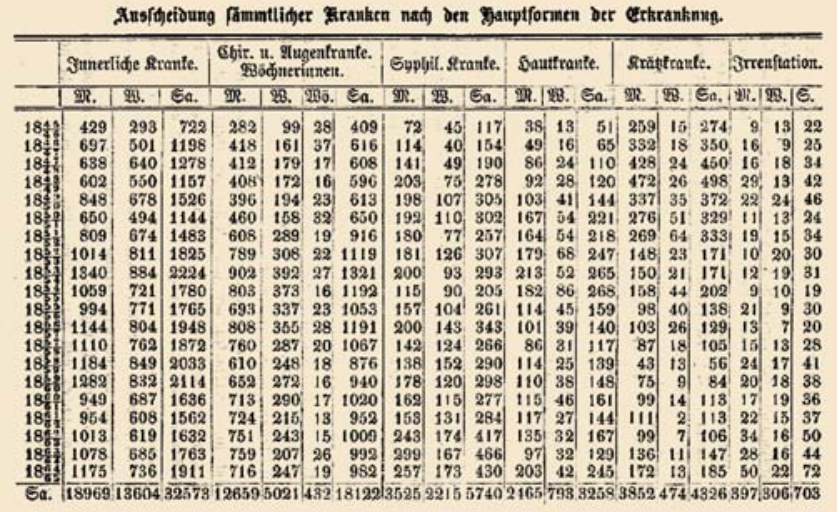

Abb. 2 Patienten der III. Abteilung: Syphilitische Kranke, Hautkranke und Krätzkranke (aus [4]). Der Anstieg der Belegungszahlen 1853/54 der Inneren Abteilung ist auf die einzige Choleraepidemie in Nürnberg 1854 zurückzuführen.

Kranke, und aus dem Sebastianspitale, der bisherigen Krankenanstalt für Syphilitische, Hautkranke und Blatternkranke, bezogen“ [4] $(\bullet$ Abb. 1).

Neben den Abteilungen für Chirurgie und Innere Medizin bestand nun die Abteilung für Haut- und Geschlechtskrankheiten sowie eine kleine Station für Geisteskrankheiten mit sechs Betten. „Es ergeben sich sonach 86 Zimmer, in denen 268 und im äußersten Nothfall 391 Kranke untergebracht werden können.“ „Die Behandlung der Kranken ist dreien ordinierenden Ärzten anvertraut, welchen zu ihrer Unterstützung, zur Führung der Journale, der Ordinationsbogen, zur Aufnahme der Kranken, zur Assistenz bei Operationen und zur Vornahme der Sectionen u.s.w. je ein junger Arzt zur Assistenz beigegeben ist" [4].

Der Abteilung für „Haut und Liebe“ standen zwölf Zimmer mit 41 bis 59 Betten für Männer und neun Zimmer mit 21 bis 35 Betten für Frauen zur Verfügung. Jeder fünfte Patient des Krankenhauses gehörte in dieser Zeit der III. Abteilung an ( $\bullet$ Abb. 2).

Als Ordinarius für die III. und IV. Abteilung (Geisteskrankheiten) wurde 1845 Dr. Ludwig Bock, der bisher das Sebastianspital betreut hatte, berufen ( Tab.1). Der zugeordnete Assistenzarzt hatte seine Wohnung im Krankenhaus. Für beide Abteilungen standen jeweils nur ein Wärter und eine Wärterin zur Verfügung [4].

\begin{tabular}{|c|c|}
\hline $1845-1865$ & Friedrich Ludwig Jakob Bock (1803 - 1865) \\
\hline $1866-1878$ & Johann Matthias Birkmeyer (gest. 1882) \\
\hline $1878-1909$ & Wilhelm Beckh (1836-1921) \\
\hline $1909-1912$ & Joseph Neuberger $(1865-1912)$ \\
\hline $1912-1922$ & Ernst Epstein $(1860-1922)$ \\
\hline $1923-1933$ & Ernst Wilhelm Nathan (1889-1981) \\
\hline $1933-1936$ & Georg Hans Birnbaum (1890 - 1948) \\
\hline $1936-1945$ & Friedrich Wilhelm Dietel (1897 - 1991) \\
\hline $1945-1964$ & Fritz Beck (geb. 1899) \\
\hline $1964-1988$ & Gerhard Weber (1923-2002) \\
\hline $1989-2008$ & Ernst Eberhard Paul (geb. 1942) \\
\hline Seit 2008 & Erwin Stefan Schultz (geb. 1968) \\
\hline
\end{tabular}

\section{Die erste kommunale Abteilung für Haut- und Geschlechtskrankheiten in Deutschland $\nabla$}

Der Beginn einer modernen stationären Dermatologie/Venerologie in Nürnberg ist somit auf das Jahr 1845 zu datieren. Damit wurde in Nürnberg eine der ersten Abteilungen für Haut- und Geschlechtskrankheiten in Deutschland geschaffen, möglicherweise die erste an einer städtischen Klinik. Frühere Gründungen von spezialisierten stationären Einrichtungen gab es lediglich an zwei Universitätskliniken: Die 1822 in Berlin geschaffene Syphilisklinik unter der Leitung von Carl Alexander Ferdinand Kluge und die 1831 in München eröffnete III. Abteilung, die Syphilitische Abteilung und Klinik unter Leitung von Johann Narr. Darüber hinaus gründete Albert Friedrich Veiel in privater Initiative bereits 1837 in Bad Cannstadt seine aus eigenen Mitteln finanzierte „Heilanstalt für Flechtenkranke“. Die nächsten Gründungen von Abteilungen für Haut- und Geschlechtskrankheiten sind erst 1874 in Dresden-Friedrichstadt und 1877 in Hamburg St. Georg dokumentiert [6].

Es mag auch daran liegen, dass Patienten mit Blattern (Pocken) im neuen Krankenhaus in der Internistischen Abteilung behandelt wurden, jedenfalls fiel nun die hohe Heilungsrate der Patienten in der Abteilung für Haut- und Geschlechtskrankheiten auf, während die Patienten mit den sog. „ekelhaften Krankheiten“ früher im Sebastianspital noch im „Institut für unheilbare Krankheiten“ untergebracht waren ( $\bullet$ Abb.3). „Es kann das günstige Heilungsresultat dieser Abtheilung nicht überraschen, da sie es in der großen Mehrzahl der Fälle nur mit sicher heilbaren Krankheiten zu thun hat. Dahin fallen an sich die Scabiösen, ein weit überwiegender Theil der Syphilitischen, und nur die Hautkranken bieten Formen dar, welche aller und jeder Medikation trotzen oder nur Besserung zulassen“ [4].

\section{Die Abtheilung für Sipphilis und Sautkrankbeiten}

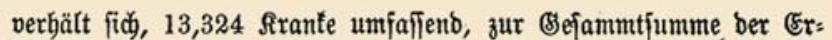
trantungen mit $20,57 \frac{0}{\sigma}$, von benen $8,86 \%$ auf bie Sypbilitifden, $6,68 \frac{0}{0}$ auf bie Siräłtranfen, $5,03 \frac{0}{0}$ auf bie \$autfranten entfallen. Sie hat folgento ङुeilungşrejultate:

\begin{tabular}{|c|c|c|c|c|}
\hline Bebeilt mu & coen & 12306 & & $92,35 \frac{0}{0}$. \\
\hline Bెebeffert. & . & 264 & . & $1,98 \frac{\circ}{0}$. \\
\hline ungebeilt & . & 153 & . & $1,14 \%$. \\
\hline Beftorben & . & 38 & . & $0,28 \%$. \\
\hline Ulebergänge & . & 563 & . & $4,22 \%$. \\
\hline & & 13324 & & $99,97$. \\
\hline
\end{tabular}

Abb. 3 Abgangsbewegung der III. Abteilung für die Jahre 1845 bis 1865 (aus [4]). 


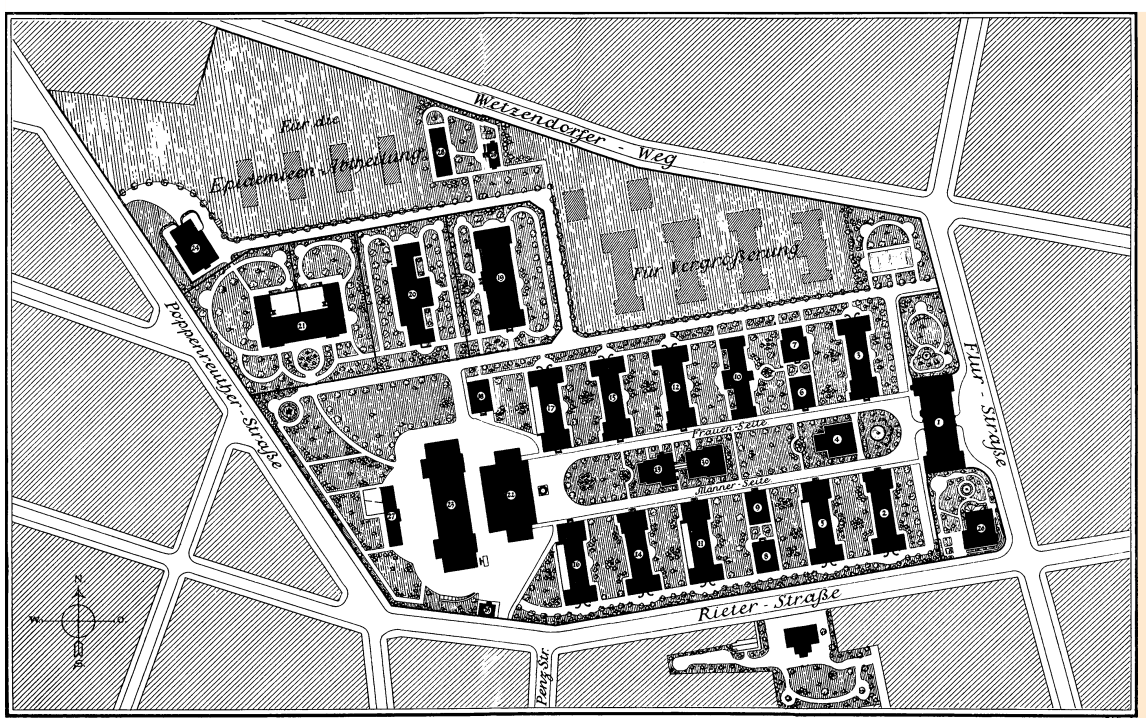

Abb. 4 Lageplan des neuen allgemeinen Krankenhauses der Stadt Nürnberg (aus [7]).

Nachdem sich Dr. Bock 1865 „nach neunzehnjähriger verdienstlicher Wirksamkeit durch ein chronischen Nervenleiden gezwungen“ sah, „seine Entlassung zu nehmen“ [4], wurde nach einem Jahr Vakanz Dr. Johann Matthias Birkmeyer, zuvor Assistent der Internistischen Abteilung, neuer Ordinarius. Dieser wurde 1878 von Dr. Wilhelm Beckh abgelöst, der seit 1862 in Nürnberg als praktischer Arzt niedergelassen war und ab $1868 \mathrm{im} \mathrm{Se-}$ bastianspital als Hausarzt in städtischem Dienst praktiziert hatte. Ab 1875 übernahmen Diakonissen der Neuendettelsauer Diakonissenanstalt größtenteils die Krankenpflege am Städtischen Krankenhaus [5].

\section{Die Dermatovenerologie als dritte klinische Fachdisziplin \\ $\nabla$}

Ein typisches Phänomen der Gründung von Abteilungen für Hautund Geschlechtskrankheiten traf auch für Nürnberg zu: Die Abteilungen für Hautkranke („Krätzige“) und Geschlechtskranke („Syphilitische“) wurden, der damaligen großen Verbreitung dieser Erkrankungen Rechnung tragend, häufig als dritte Abteilung neben der Inneren Medizin und der Chirurgie erschaffen. Zuvor hatten die Hautkrankheiten traditionell zur Inneren Medizin, die Geschlechtskrankheiten zur Äußeren Medizin gehört [6]. Die Hautklinik im Klinikum Nürnberg trägt bis heute, nach 165 Jahren, als Krankenhausabteilung die Bezeichnung Kh/III.

Ebenso typisch auch für andere damalige Neugründungen ist, dass die leitenden Ärzte häufig mehrere Abteilungen führten, so leitete Kluge in Berlin von 1822 bis 1844 neben der Syphilisklinik auch die Abteilung für Geburtshilfe [6]. Auch in Nürnberg wurden die Abteilungen für Haut- und Geschlechtskranke und für Geisteskranke von 1845 bis 1909 von einem Ordinarius, später „Oberarzt“, geleitet.

Aufgrund ständig steigender Patientenzahlen waren die räumlichen und hygienischen Zustände im Alten Städtischen Krankenhaus schon bald unzureichend. Die Bevölkerung der Stadt Nürnberg war von 1845/46 mit 47390 Einwohnern auf 65708 Einwohner bereits im Jahr 1863/64 angestiegen [4]. Im Jahr 1897 lebten schon 172000 Menschen in Nürnberg. Die Anzahl stationärer Patienten hatte sich von $1530 \mathrm{im}$ Jahr der Eröffnung auf $4600 \mathrm{im} \mathrm{Jahr}$ 1886 verdreifacht [1] und auf 5800 im Jahr 1896 beinahe vervierfacht [7]. Nach mehrfachen baulichen Erweiterungen mit Pavil- lonbauten und Baracken im südlichen Gartengelände war ein weiterer Aus- oder Anbau aus Platzgründen und wegen der ungenügenden Abwasserentsorgung nicht mehr möglich.

\section{Das Neue Städtische Krankenhaus an der Flurstraße $\nabla$}

1889 beantragte die Krankenhauskommission, das Städtische Krankenhaus an anderer Stelle neu zu erbauen, nach dem Entschluss der Stadt Nürnberg für einen Neubau im Stadtteil St. Johannis im Nordwesten der Stadt konnte nach vierjähriger Bauzeit am 5. 9.1897 das im Pavillonstil errichtete neue Städtische Krankenhaus an der Flurstraße mit 30 Gebäuden und 761 Betten eröffnet werden. Als Vorbild dienten die neu errichteten Krankenhäuser in Berlin „Am Urban“ und in Hamburg-Eppendorf [1]. Die chirurgische Klinik (Abteilung I) des Neuen Städtischen Krankenhauses umfasste fünf Gebäude mit insgesamt 204 Betten, die medizinische Klinik (Abteilung II) ebenfalls fünf Gebäude mit 332 Betten. Auf dem Lageplan ( $\bullet$ Abb.4) erkennt man die Ausrichtung der Bettenhäuser entlang der zentralen Achse. Männer und Frauen waren getrennt untergebracht mit der Männerseite im Süden und der Frauenseite im Norden der Mittelanlage, in der das Operationshaus, das Badehaus und das Gebäude für Heilgymnastik errichtet waren [7].

Die Abteilung für Hautkrankheiten und Syphilis (Abteilung III) war, außerhalb der beiden Pavillonreihen im Nordwesten des Geländes, in den Gebäuden 19 (eingeschossiger Krankenbau mit 60 Betten für Männer) und 20 (zweigeschossiger Bau mit 78 Betten für Frauen) ebenfalls nach Geschlecht der Patienten getrennt untergebracht ( Abb. 5 und 6). „Bei der dritten Abteilung ist jedes Gebäude mit seinem dazugehörigen Garten der sicheren Abtrennung wegen mit einer Mauer umgeben, die Abstände der einzelnen Bauten mussten infolge dessen hier größer genommen werden, durchschnittlich 30 m“ [7]. Ein weiterer kleiner eingeschossiger Krankenbau (Bau 18) diente als Isolierbau und enthielt weitere acht Betten, sodass zum Erbauungszeitpunkt im Jahre 1897 die Abteilung für Hautkrankheiten und Syphilis 146 Betten umfasste. Die Abteilung IIla für Geisteskranke war, ebenfalls von eigenen Mauern umschlossen, in Bau 21 mit je 16 Betten für Frauen und Männer untergebracht. Als Oberarzt der Abteilungen III und IIIa amtierte weiterhin der 1895 zum königlich bayerischen Hofrat ernannte Dr. Wilhelm Beckh. 


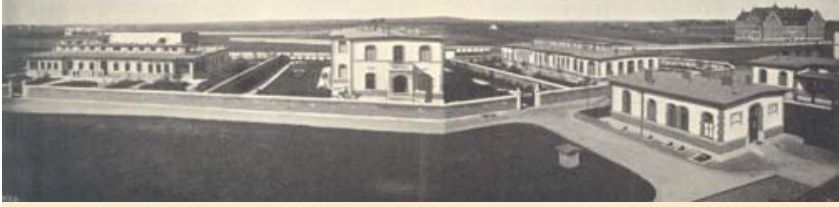

Abb. 5 Auf der zusammengefügten Fotografie aus südlicher Richtung erkennt man links den Bau 21 der Abteilung IIla für Geisteskranke, mittig und rechts die Bauten 20, 19 und 18 der Abteilung III für Haut- und Geschlechtskranke. Im Westen, Norden und Osten grenzen im Erbauungsjahr 1897 Wiesen und Felder an das Klinikgelände (aus [7]).

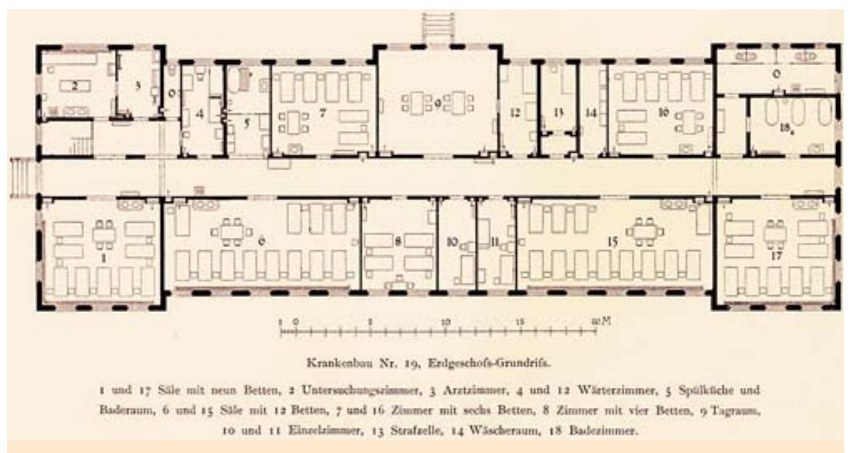

Abb. 6 Grundriss des eingeschossigen Korridorbaus für Männer (aus [7]). Die Einrichtung einer Strafzelle deutet auf die strenge Disziplin im Krankenhaus der damaligen Zeit hin. Patienten wurden für die Zeit ihrer Einweisung zu Anstaltsinsassen mit Anstaltskleidung und strengen Regeln.

Insgesamt wurden 761 Betten plus die Pathologie von zwölf Ärzten betreut. Der gesamte Pflegedienst inklusive Bademeister und Sektionsdiener umfasste 42 Personen [7]. Die Krankenpflege in der Abteilung für Haut- und Geschlechtskranke wurde bereits seit 1875 bei den weiblichen Patientinnen von den evangelischen Diakonissen der Diakonie Neuendettelsau, bei den männlichen Patienten (und in der Abteilung für Geisteskranke) von freien Schwestern und Pflegern ausgeübt. „Auf den männlichen Abteilungen finden seit dem Jahre 1890 im Krankendienste junge Männer aus der evangelischen Diakonenbildungs-Anstalt Verwendung, namentlich zur Vornahme gewisser dem weiblichen Pflegepersonal nicht zuzumutender Handreichungen“ [7]. Das Pflegepersonal wohnte in den betreffenden Krankengebäuden, ebenso wie der Assistenzarzt, der unverheiratet sein musste.

Als Hofrat Beckh 1909 nach über 30-jähriger Dienstzeit in den Ruhestand ging, wurden die Abteilungen für Haut- und Geschlechtskranke und für Geisteskranke verselbständigt, die Klinik für Geisteskranke wurde zur Abteilung IV mit 43 Betten [5], neuer Oberarzt der Abteilung III wurde erstmals ein Facharzt für Haut- und Geschlechtskrankheiten, Dr. Joseph Neuberger (1865-1912), der allerdings nach drei Jahren im Alter von nur 47 Jahren starb.

1914 hatte sich die Gesamtbettenzahl des Krankenhauses gegenüber der Eröffnung 1897 mit 1300 Betten beinahe verdoppelt [5]. Im Zuge der ständigen Erweiterung der Krankenbauten aufgrund stetig steigender Patientenzahlen wurde bereits im Jahre 1912 auch der Bau 19 für haut- und geschlechtskranke Männer um eine Etage aufgestockt, die Bettenzahl im Bau 20 war auf 158 Betten für Frauen angestiegen. Im November des gleichen Jahres wurde Dr. Ernst Epstein (1860 - 1922) zum Oberarzt berufen. Er stammte aus Breslau, hatte sich 1887 als erster Facharzt für Hautund Geschlechtskrankheiten in Nürnberg [3] niedergelassen und stets auch wissenschaftlich betätigt [5].

\section{Die Geschlechtskrankheiten als Schwerpunkt der stationären Versorgung \\ $\nabla$}

Ab 1917 betrieb die Landesversicherungsanstalt Mittelfranken eine eigene Beratungsstelle für Geschlechtskrankheiten, in der eine kostenlose und streng vertrauliche Beratung durchgeführt wurde. Diese Beratungen wurden von Epstein neben seiner Oberarzttätigkeit am Städtischen Krankenhaus durchgeführt. Nach dem Ersten Weltkrieg nahmen die Geschlechtskrankheiten, insbesondere die Syphilis, auch in Nürnberg rapide zu. „Der verlorene Krieg, die überstürzte Demobilmachung, das ungeheure Wohnungselend und die erschütterten ethischen Grundlagen unseres Volkes sind die Ursachen der Ausbreitung dieser Seuche“, schrieb der administrative Leiter des 1920 in Nürnberg neu gegründeten städtischen Gesundheitsamtes Dr. Robert Plank [8].

1923 wurde Prof. Ernst Wilhelm Nathan als neuer Oberarzt der Abteilung für Haut- und Geschlechtskrankheiten berufen. Nathan hatte in Gießen, München und Berlin studiert. Nach Staatsexamen und Promotion wurde er 1913/14 Assistent an der experimentell-biologischen Abteilung des Instituts für experimentelle Therapie in Frankfurt am Main unter der Leitung von Paul Ehrlich (1854-1915), der 1910 das Salvarsan und 1912 das Neosalvarsan entwickelt und damit die erste gezielte Therapie der Syphilis eingeführt hatte. Anschließend absolvierte Nathan die klinische Ausbildung als Assistent und Oberarzt an der ab 1914 universitären Hautklinik in Frankfurt-Sachsenhausen als Schüler von Karl Herxheimer (1861 - 1942) [9,10]. Nathan wurde 1918 in Frankfurt habilitiert und 1922 zum außerordentlichen Professor ernannt. Bevor er 1923 die Leitung der Nürnberger Klinik übernahm, hatte er an die 50 Publikationen verfasst, etliche gemeinsam mit Herxheimer, die sich vor allem mit Nachweisverfahren der Syphilis wie der Wassermann'schen Reaktion, anderen Komplementphänomenen, Nebenwirkungen und Weiterentwicklungen des Salvarsan sowie dermatophysiologischen und dermatotherapeutischen Themen beschäftigten.

Außer der Klinikleitung in Nürnberg übernahm Nathan 1923 von Epstein auch die Tätigkeit in der Beratungsstelle für Geschlechtskrankheiten und wurde zugleich Leiter der neu geschaffenen Poliklinik für Geschlechtskranke. Zusätzlich war Nathan auch für die Untersuchung der Prostituierten zuständig [5]. „Nachdem Gesundheitsamt, Beratungsstelle, Poliklinik und Krankenhaus durch einen lückenlos organisierten Meldedienst in engster, gegenseitiger Fühlung stehen, ist ein engmaschiges Netz über alle diesen Stellen bekannt werdenden Ansteckungsquellen geworfen, das durch einen gutfunktionierenden Nachrichtendienst mit den auswärtigen Verwaltungsstellen und Einwohnerämtern noch ergänzt wird“, berichtete Plank 1924 [8].

Die Zahl der Beratungen und Behandlungen nahm stetig zu, sodass die Zusatzfunktionen von Nathan nicht mehr nebenamtlich zu leisten waren. Daher stellte die Stadt Nürnberg 1929 einen hauptamtlichen Geschlechtskrankenfürsorgearzt ein, Dr. Anton Munk. Auch die stationäre Belegung mit Geschlechtskranken nahm weiter zu, sodass 1929 die venerologische Abteilung von 100 auf 142 Betten ausgebaut wurde. Auf Antrag von Nathan wurde im gleichen Jahr eine eigene Krankenhausfürsorgerin für die weiblichen Geschlechtskranken eingestellt, zu deren Aufgaben die wirtschaftliche und gesundheitliche Fürsorge, Fragen der Berufsumschulung, der Wohnung nach der Entlassung und die Beschäftigung der Kranken während des Krankenhausaufenthaltes zählten [11]. 


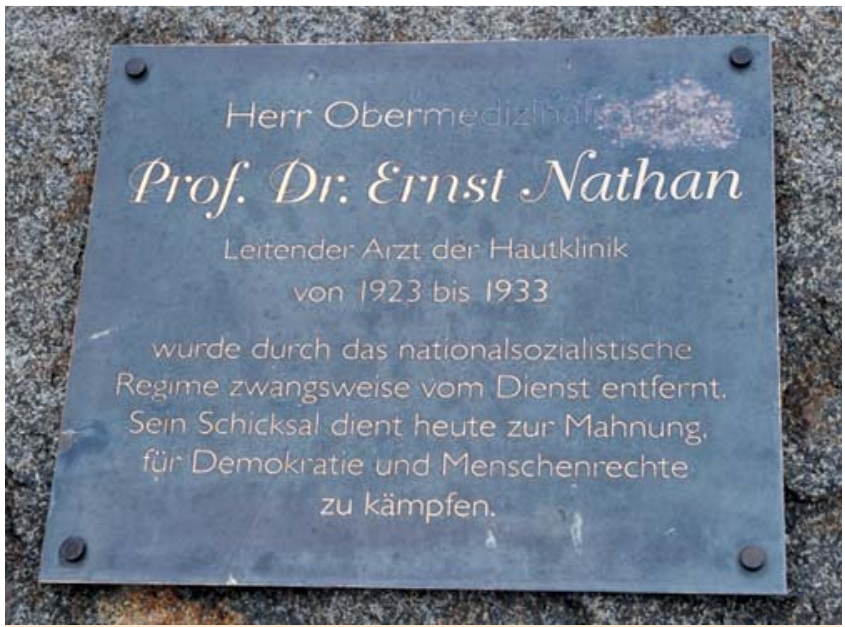

Abb. 7 Gedenktafel für Prof. Ernst Nathan.

\section{Der Chefarzt der Hautklinik als Opfer der Judenverfolgung \\ $\nabla$}

Bereits im Oktober 1923 hatte der spätere Gauleiter der NSDAP für Mittelfranken Julius Streicher in seinem „Stürmer“ [12] gedroht, die „schandbare Verknoblauchung“ und „Verjudung des Krankenhauses“ zu beseitigen: „Einen Trost haben wir noch: Die Herren Dr. Veithe und Dr. Nathane werden an dem Tag aus ihrem neuen Amtsbereich herausgeholt, an welchem die Hakenkreuzfahne auf dem Luppe-Rathaus weht."

Schleicher sollte Recht behalten, denn Ende März 1933 wurde Nathan als Chefarzt der Hautklinik suspendiert und kraft „Gesetzes zur Wiederherstellung des Berufsbeamtentums" im September 1933 ohne Ruhegehalt entlassen [13]. Zunächst konnte Nathan in seiner Wohnung weiter praktizieren, nach dem Approbationsentzug jüdischer Ärzte durch die Nationalsozialisten vom 30.9.1938 war jedoch auch dies nicht mehr möglich. Nathan konnte am 29.3.1939 nach New York emigrieren, wo er nach langjähriger ärztlicher Tätigkeit, u.a. einer Lehrtätigkeit an der Skin-and-Cancer-Unit der New York-University, 1981 starb [14]. 1995 wurde auf Initiative des damaligen Nürnberger Gesundheitsreferenten Klaus-Peter Murawski eine Gedenktafel für Prof. Nathan im Innenhof der Hautklinik aufgestellt, die sich mittlerweile vor dem neuen Eingang der Hautklinik vor Haus 16 befindet ( $\odot$ Abb. 7). 1998 wurde auf Initiative der Nürnberger Ortsgruppe der IPPNW ein Teilabschnitt der Flurstraße, und somit die Adresse des Klinikums Nord, in Prof.-Ernst-Nathan-Straße umbenannt [5].

\section{Die Hautklinik im Nationalsozialismus und im Zweiten Weltkrieg \\ $\nabla$}

Bis November 1933 leitete Dr. Wilhelm Guttenberg kommissarisch die Hautklinik, dann wurde Prof. Georg Hans Birnbaum neuer Chefarzt. Birnbaum erhielt die Approbation 1914 in Berlin, wurde 1918 in Rostock promoviert und arbeitete als Assistent und Oberarzt von 1919 bis 1927 bei Prof. Karl Zieler (1874-

\footnotetext{
${ }^{1}$ Dr. Adolf Veith war 1923 neuer leitender Arzt der Röntgenabteilung geworden. Dr. Hermann Luppe war Gründungmitglied der liberalen DDP und seit 1920 Oberbürgermeister von Nürnberg. Er wurde im März 1933 nach der Machtübernahme der Nationalsozialisten verhaftet und zum Rücktritt gezwungen.
}

1945) in Würzburg, wo er 1924 habilitiert wurde. Er war bereits von 1927 bis 1933 Chefarzt der Hautklinik in Dortmund gewesen, bevor er die Stelle in Nürnberg antrat. Die Tätigkeit in Nürnberg übte er nur bis Ende 1936 aus, da er einen Ruf an den Lehrstuhl der Universität Königsberg erhielt und ab Januar 1937 dort Direktor der Universitätsklinik und Poliklinik für Haut- und Geschlechtskrankheiten wurde $[15,16]^{2}$

Ab 1934 wurde die Untersuchung geschlechtskranker Frauen, insbesondere der Prostituierten („Personen mit häufig wechselndem Geschlechtsverkehr“) direkt im Städtischen Krankenhaus durchgeführt [11]. Zu diesem Zweck wurde der bislang für Epidemien vorbehaltene Bau 28 umgebaut. Nun konnte die Überwachung und Behandlung der Frauen zentral im Krankenhaus erfolgen [5].

Die freigewordene Chefarzt-Position erhielt ab Dezember 1936 Prof. Friedrich Wilhelm Dietel. Dieser war Nürnberger und hatte in Erlangen studiert. Nach der Approbation 1922 und der Promotion 1923 arbeitete er als Assistent an der Inneren Abteilung in Hagen und ab 1923 an der Universitäts-Hautklinik in Erlangen unter Leo Hauck (1874-1945). Im Jahre 1926 hielt er sich für zwei Monate als Gast an der Breslauer Hautklinik von Josef Jadassohn (1863 - 1936) auf. In Erlangen wurde er 1928 habilitiert und 1934 zum außerordentlichen Professor ernannt $[18,19]$.

Neben der Leitung der Nürnberger Hautklinik übte er seine Lehrtätigkeit an der Universität Erlangen bis 1945 weiter aus. „So verstand er es auch, seine Hörer zum wissenschaftlichen Arbeiten anzuregen, wovon die große Zahl der unter seiner Anleitung gefertigten Dissertationen ein beredtes Zeugnis ablegen. Nach 1945, ohne die ihn voll ausfüllende klinische Tätigkeit, übte er zunächst in Lauf, alsbald wieder in Nürnberg eine große, angesehene, praktische dermatologische Tätigkeit aus“, heißt es in der Dermatologischen Wochenschrift zum 60.Geburtstag Dietels $1957[20]^{3}$

Nach dem deutschen Überfall auf Polen und dem Beginn des Zweiten Weltkriegs kam es zu einer zunehmenden Einweisung von ausländischen Kriegsgefangenen und Zwangsarbeitern in das Städtische Klinikum. Davon war besonders die Hautklinik betroffen, da bei diesen Patienten häufig ein Lausbefall oder Krätze festgestellt wurde. Zunächst konnte die Behandlung in den normalen Klinikbetrieb integriert werden. Die Krätzeerkrankungen nahmen jedoch auch bei der einheimischen Bevölkerung stark zu. Ab 1942/43 wurden in der nordwestlichen Ecke des Klinikgeländes fünf „Ausländer-Krankenbaracken“ aufgestellt, in denen die Zwangsarbeiter ab Frühjahr 1943, isoliert von den anderen Kranken, untergebracht wurden [5].

Ab März 1943 bis zum Kriegsende wurden durch die Luftangriffe der Alliierten von den 35 Gebäuden des Klinikums 12 so gut wie völlig zerstört, weitere 16 stark beschädigt [21]. Die Hautklinik war gemeinsam mit der Inneren Medizin und der Neurologie nach Ansbach ausgelagert worden. Der übrige Klinikbetrieb wurde provisorisch im Hochbunker, der zwischen 1940 und 1943 erbaut wurde, und einem Tiefbunker weitergeführt [5]. Bei einem Bombenangriff kamen im Luftschutzraum in Bau 19 mehrere Menschen ums Leben [22].

\footnotetext{
${ }^{2}$ Diese Berufung Birnbaums als „Beispiel für die höhere Bewertung politischer Zuverlässigkeit als wissenschaftlicher Verdienste“ wird von Scholz et al. [17] ausführlich erörtert.

3 Als Mitglied der SA, des NSDÄB und der NSDAP wurde Dietel 1945 von der Militärregierung im Zuge der Entnazifizierung entlassen, in den Spruchkammerverfahren wurde er 1946 als „Mitläufer“ eingestuft [19].
} 
Hautklinik des Allgemeinen Krankenhauses Nürnberg

\begin{tabular}{|c|c|c|c|c|c|c|}
\hline \multicolumn{5}{|c|}{$\begin{array}{l}\text { Lues-Krankenziffern in den Jahren } 1932 \text { bis } 1938 \text {, sowie } 1946 \\
\text { und 1. Halbjahr } 1947\end{array}$} & \multicolumn{2}{|c|}{$\begin{array}{l}\text { Gonorrhoe- Krankenziffern } \\
\text { in den Jahren } 1932 \text { bis } 1938, \\
\text { sowie } 1946 \text { und 1. Halbjahr } 1947\end{array}$} \\
\hline Jahr & Lues I und II & Lues III & Lues latens & Gesamtzahl & Jahr & Gesamtzahl \\
\hline 1932 & $\begin{array}{r}28(\mathrm{M}=12) \\
(\mathrm{F}=16)\end{array}$ & 0 & $\begin{array}{r}55(\mathrm{M}=18) \\
(\mathrm{F}=37)\end{array}$ & 83 & 1932 & $\begin{array}{r}373(\mathrm{M}=138) \\
(\mathrm{F}=235)\end{array}$ \\
\hline 1933 & $\begin{array}{r}32(\mathrm{M}=21) \\
(\mathrm{F}=11)\end{array}$ & $\begin{array}{r}1(\mathrm{M}=0) \\
(\mathrm{F}=1)\end{array}$ & $\begin{array}{r}17(\mathrm{M}=4) \\
(\mathrm{F}=13)\end{array}$ & 50 & 1933 & $\begin{array}{r}309(\mathrm{M}=94) \\
(\mathrm{F}=215)\end{array}$ \\
\hline 1934 & $\begin{array}{r}19(\mathrm{M}=6) \\
(\mathrm{F}=13)\end{array}$ & $\begin{array}{r}6(\mathrm{MI}=2) \\
(\mathrm{F}=4)\end{array}$ & $\begin{array}{r}43(\mathrm{M}=12) \\
(\mathrm{F}=31)\end{array}$ & 68 & 1934 & $\begin{array}{r}310(\mathrm{M}=99) \\
(\mathrm{F}=211)\end{array}$ \\
\hline 1935 & $\begin{array}{r}19(\mathrm{M}=7) \\
(\mathrm{F}=12)\end{array}$ & 0 & $\begin{array}{r}28(\mathrm{M}=3) \\
(\mathrm{F}=25)\end{array}$ & 47 & 1935 & $\begin{array}{r}285(\mathrm{M}=77) \\
(\mathrm{F}=208)\end{array}$ \\
\hline 1936 & $\begin{array}{r}18(\mathrm{M}=4) \\
(\mathrm{F}=14)\end{array}$ & $\begin{array}{r}2(\mathrm{M}=1) \\
(\mathrm{F}=1)\end{array}$ & $\begin{array}{r}29(\mathrm{M}=4) \\
(\mathrm{F}=25)\end{array}$ & 49 & 1936 & $\begin{array}{r}265(\mathrm{M}=77) \\
(\mathrm{F}=188)\end{array}$ \\
\hline 1937 & $\begin{array}{r}29(\mathrm{M}=8) \\
(\mathrm{F}=21)\end{array}$ & $\begin{array}{r}6(\mathrm{M}=2) \\
(\mathrm{F}=4)\end{array}$ & $\begin{array}{r}14(\mathrm{M}=2) \\
(\mathrm{F}=12)\end{array}$ & 49 & 1937 & $\begin{array}{r}284(\mathrm{M}=67) \\
(\mathrm{F}=217)\end{array}$ \\
\hline 1938 & $\begin{array}{r}29(\mathrm{M}=17) \\
(\mathrm{F}=12)\end{array}$ & $\begin{array}{r}3(\mathrm{M}=3) \\
(\mathrm{F}=0)\end{array}$ & $\begin{aligned} 34(\mathrm{M}=10) \\
(\mathrm{F}=24)\end{aligned}$ & 66 & 1938 & $\begin{array}{r}279(\mathrm{M}=66) \\
(\mathrm{F}=213)\end{array}$ \\
\hline 1946 & $\begin{array}{r}870(\mathrm{M}=256) \\
(\mathrm{F}=614)\end{array}$ & 0 & $\begin{array}{r}350(\mathrm{M}=31) \\
(\mathrm{F}=319)\end{array}$ & 1220 & 1946 & $\begin{array}{r}4349(\mathrm{M}=1359) \\
(\mathrm{F}=2990)\end{array}$ \\
\hline $\begin{array}{c}1947 \\
\text { (1. Halbj.) }\end{array}$ & $\begin{aligned} 532(\mathrm{M} & =150) \\
(\mathrm{F} & =382)\end{aligned}$ & $\begin{aligned} 2(\mathrm{M}=1) \\
(\mathrm{F}=1)\end{aligned}$ & $\begin{array}{r}314 \quad(\mathrm{M}=26) \\
(\mathrm{F}=288)\end{array}$ & 848 & $\begin{array}{c}1947 \\
\text { (1. Halbj.) }\end{array}$ & $\begin{array}{r}1566(\mathrm{M}=357) \\
(\mathrm{F}=1209)\end{array}$ \\
\hline
\end{tabular}

Abb. 8 Waren 1938 „nur“ 66 Fälle von Lues und 279 Fälle von Gonorrhoe in der Hautklinik behandelt worden, stiegen diese im Jahr 1946 auf 1220 bzw. 4349 Fälle an (aus [21]).

\section{Seuchenbekämpfung und Wiederaufbau \\ $\nabla$}

1945 hatte Dr. Fritz Beck zunächst vertretungsweise die Leitung der Hautklinik übernommen. Beck hatte in Würzburg und München studiert, begann 1925 eine chirurgische und allgemeinmedizinische Ausbildung in Würzburg, bevor er sich 1927 der dermatologischen Ausbildung zuwandte, die er zunächst in Würzburg unter Karl Zieler, ab 1930 in Dortmund unter Georg Birnbaum absolvierte. 1934/35 arbeitete er am Rudolf-Virchow-Krankenhaus in Berlin bei Heinrich Löhe (1877 - 1961), ab 1935 an der Hautklinik Nürnberg unter Birnbaum und Dietel [15]. Er wurde 1945 zunächst kommissarischer Leiter der Hautklinik und sollte diese doch für beinahe 20 Jahre führen. „Die schweren Zerstörungen der Hautklinik in den letzten Monaten des 2. Weltkrieges stellten den damaligen Klinikvorstand vor schwere Probleme. Diese resultierten aus einem bis 1946 und zur Wende 1947 anschwellenden Strom von Geschlechtskrankheiten, Krätzekranken und mit Infektionen der Haut behafteten Patienten“ [23].

In den ersten Nachkriegsmonaten verbreiteten sich aufgrund der mangelhaften und unhygienischen Lebensbedingungen in der stark zerstörten Stadt ansteckende Erkrankungen, unter anderem Kopfläuse und Krätze, sowohl in den Flüchtlingslagern als auch bei der einheimischen Bevölkerung. Im Jahr 1946 wurden noch 1715 Fälle von Krätze registriert, 1951 nur noch 15 [5]. Auch die Geschlechtskrankheiten nahmen in den Nachkriegsjahren wieder rasant zu, „... es nahm der Kampf gegen die unheimlich sich ausbreitende Seuche der Geschlechtskrankheiten seinen Anfang“" [21].

„Von den 1946 gezählten 6967 Patienten (der Hautklinik) waren allein 5395 geschlechtskrank. Ihre Unterbringung stieß auf erhebliche Schwierigkeiten, da die Gesamtbettenzahl der Städtischen Krankenanstalten durch Kriegseinflüsse stark reduziert und somit keine Ausweichmöglichkeit gegeben war. Überbelegung, vorzeitige Entlassung und ambulante Versorgung waren unerlässlich“ [23] ( Abb. 8).

Die Nachkriegsjahre stellten für das gesamte Krankenhaus durch die Kriegszerstörungen eine enorme bauliche und organisatorische Herausforderung dar, alle Kraft wurde in den Wiederaufbau gesteckt ( $\bullet$ Abb.9 und 10), während mit großem Improvisationstalent versucht wurde, des sprunghaft ansteigenden Patientenzustroms Herr zu werden.

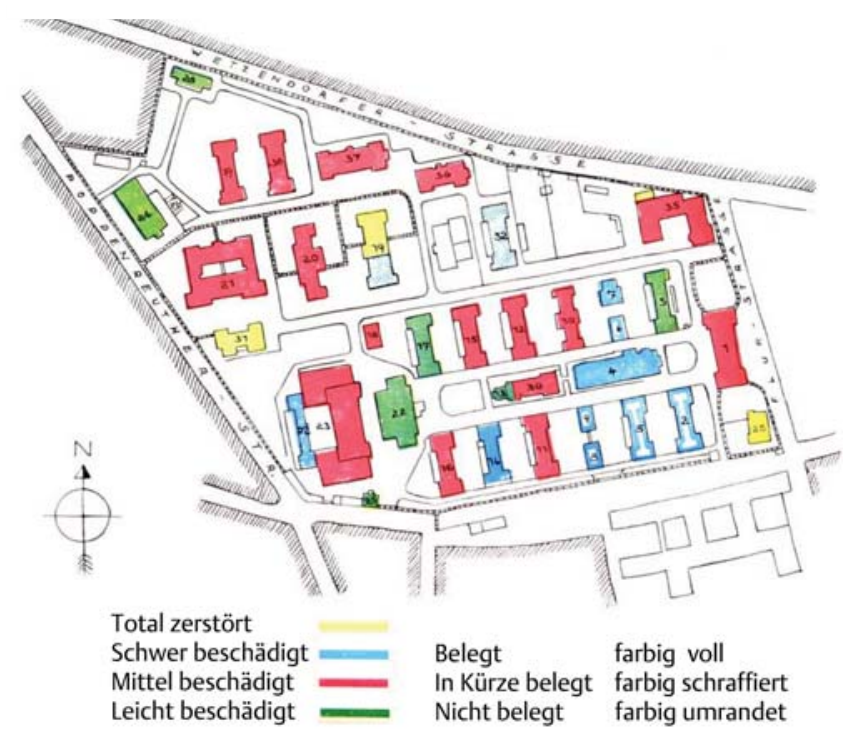

Abb.9 Nach Kriegsende war Bau 19 zum größeren Teil total zerstört, zum kleineren Teil schwer beschädigt, Bau 20 war mittel beschädigt (aus [21]).

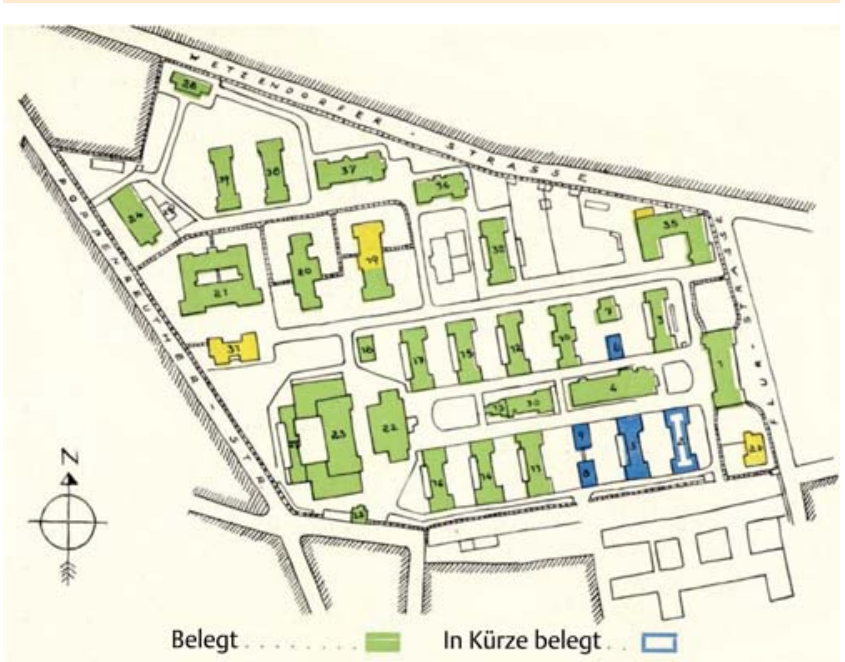

Nicht belegt .... Total zerstört

Abb. 10 Bis September 1947 wurden der schwer beschädigte Teil von Bau 19 und der gesamte Bau 20 wieder aufgebaut und konnten belegt werden (aus [21]). 


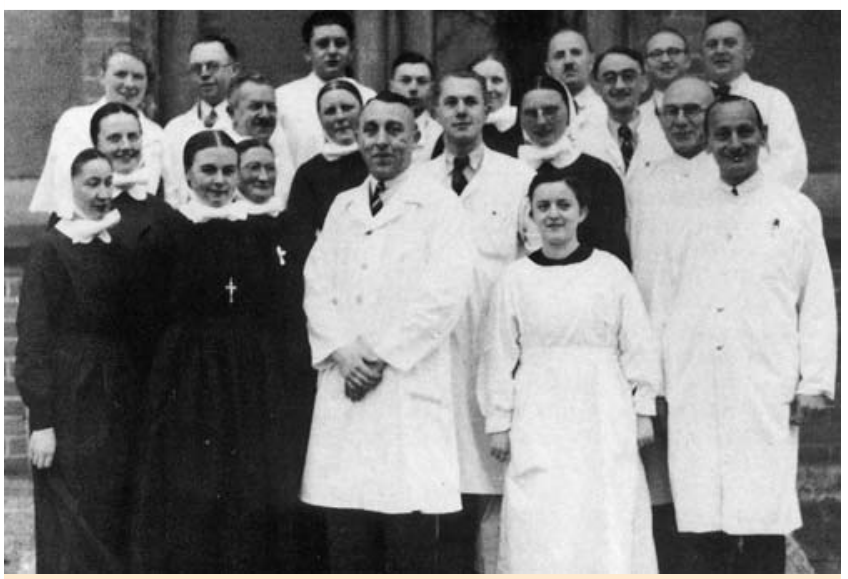

Abb. 11 Medizinaldirektor Dr. Fritz Beck im Kreise seiner Mitarbeiter ca. 1960. Die Neuendettelsauer Diakonissen bilden noch die Mehrheit des Pflegepersonals (aus [5]).

1947 umfasste die Hautklinik 426 Betten, verteilt auf die Bauten 14, 16, 19, 20 und 28. Die Hautklinik stellte in dieser Zeit die größte Abteilung des Krankenhauses, die Innere Medizin war inzwischen in die 1. und 2. Medizinische Klinik unterteilt mit 406 bzw. 277 Betten. Neben Beck waren 7 Assistenzärzte, 3 Gastärzte und ein Medizinalpraktikant für die Versorgung der Kranken zuständig, das nichtärztliche Personal umfasste 58 Personen [21] (- Abb. 11).

Unter dem Eindruck der großen Patientenzahl wurden schon bald nach Kriegsende Planungen für eine Erweiterung der Hautklinik vorgenommen, sodass 1953 auch der zerstörte Teil von Bau 19 wiederaufgebaut wurde und durch einen Querflügel die Bauten 19 und 20 U-förmig verbunden wurden. 1954 konnte die Hautklinik mit sieben Männer-, Frauen- und Kinderstationen sowie Funktionsräumen den Bau 19 beziehen. Es waren Ein-, Zwei- und Sechsbettzimmer, aber auch Säle mit 12 bis 14 Betten vorhanden. Die geschlechtskranken Frauen wurden in einer geschlossenen Abteilung im Hochbunker untergebracht [23], sie wurden täglich zur Chefvisite nach Bau 19 und wieder zurückgeführt [22].

„Die Normalisierung im zahlenmäßigen Verhältnis dermatologisch und venerologisch Kranker erfolgte mit einer jährlichen Verminderung der Geschlechtskrankenzahl von ca. 30\% relativ schnell. So wurden im Jahre 1948 noch 2400, 1951 nur noch 643 Geschlechtskranke und 1971 lediglich 70 solcher Patienten aufgenommen. Die Zahl der Hautkranken zeigte von 1947 bis 1963 mit etwa 1150 Patienten jährlich ein relativ stabiles Zahlenverhältnis.“ [23]. Aufgrund des sinkenden Bedarfs an stationären Betten wurden diese nach und nach reduziert. 1960 verfügte die Hautklinik über 220 Betten.

\section{Die Ära Gerhard Weber \\ $\nabla$}

Als Beck 1964 nach Erreichen der Altersgrenze ausschied, wurde Prof. Dr. Gerhard Weber neuer Chefarzt der Nürnberger Hautklinik. Er war ab 1949 Assistent an der Universitäts-Hautklinik in Mainz unter Egon Keining (1892-1971) gewesen und hatte hier seine Facharztausbildung absolviert. Es folgten Habilitation und Professur. Gemeinsam mit Otto Braun-Falco (geb. 1922), mit dem zusammen er eine Vielzahl an Publikationen, insbesondere zu Hyaluronidase, verfasste, war er in Mainz Oberarzt, zuletzt seit 1961 unter Günter W. Korting (1919-1994) [24].

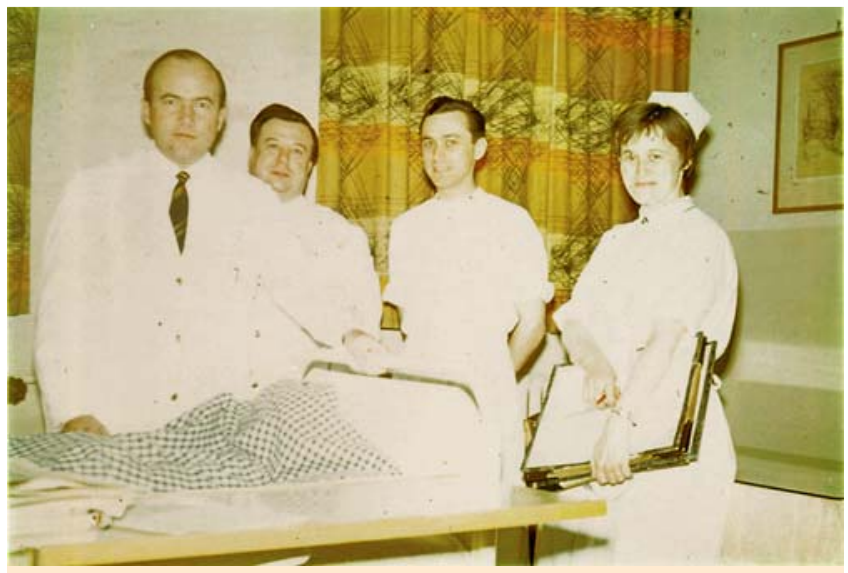

Abb. 12 Prof. Weber bei der Chefvisite Mitte der 1960er-Jahre, gemeinsam mit Assistenzarzt Dr. Vogel, Pfleger Ansbert Alex und Schwester Annemarie Popp. Die Hautklinik hatte spezielle Bettwäsche mit blau-weißem Muster, das gegen Verfärbungen durch Teer, Cignolin und Farbstoffe weniger empfindlich war. Die „Anstaltskleidung“ für die Patienten war in der Hautklinik blau-weiß gestreift [22].

„Der großzügige Bau der Hautklinik erlaubte es, dass seit der Amtsübernahme von Prof. Dr. Weber im Jahre 1964 eine Reihe organisatorischer und struktureller Änderungen durchgeführt werden konnten, deren wichtigstes Ergebnis ist, dass die Patientenzahl seitdem unaufhörlich anstieg und im Jahre $1971 \mathrm{um}$ rund 50\% höher lag als im Durchschnitt der Jahre 1954 bis 1964“ [23]. 1965 stellte die Neuendettelsauer Diakonissen-Anstalt die seit Jahrzehnten bewährte Entsendung ihrer Schwestern aus Mangel an Nachwuchs ein. Bis zu deren Ersatz durch Schwestern des Roten Kreuzes und vor allem durch freie Schwestern und Pfleger mussten zwei Stationen der Hautklinik vorübergehend außer Betrieb genommen werden. Im weiteren Verlauf wurde auch die in den Hochbunker ausgelagerte geschlossene venerische Station für Frauen zurück in die Hautklinik integriert. „Durch geringe bauliche Veränderungen wurde ihre ebenfalls sichere, aber zugleich menschliche Unterbringung erreicht“ [23]. Im „GO-Zimmer" fanden allerdings weiterhin die polizeilichen Vorführungen von aufgegriffenen Personen, bei denen der Verdacht auf eine Geschlechtskrankheit bestand, statt. Bei positivem GO-Abstrich bzw. klinischem Verdacht auf Vorliegen einer Geschlechtskrankheit erfolgte die stationäre Aufnahme.

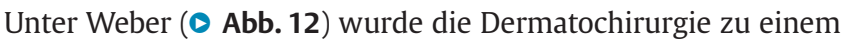
der Schwerpunkte der Nürnberger Hautklinik. Dies betraf zum einen Tumoroperationen, zum anderen plastische Defektdeckungen durch Spalthauttransplantation nach Verbrennungen: Am Anfang dieser Entwicklung stand nur ein einziges Dermatom der Fa. Stryker zur Verfügung, das im Reparaturfall extra in die USA geschickt werden musste [25], aber „nach mehrjährigen technischen und organisatorischen Vorarbeiten wurde der Ausbau einer Intensivpflegestation für Verbrennungskranke im Jahr 1968 genehmigt und ausgeführt“ [23]. Im Oktober 1977 wurde unter Federführung Webers in Nürnberg die Vereinigung für operative Dermatologie (VOD) gegründet [26].

Ein anderer wissenschaftlicher Schwerpunkt war die Erforschung der UV-Therapie nach vorangegangener Lichtsensibilisierung der Haut zur Behandlung der Vitiligo, später auch der Psoriasis. Weber stand hierbei in engem Kontakt mit dem Pionier der PUVA-Therapie Prof. Abdel M. El Mofty [25]. „Die außergewöhnliche Zusammensetzung des Krankengutes hinsichtlich Form und Schwere der Hauterkrankungen schaffte eine anderen- 
orts nur selten anzutreffende Basis für wissenschaftliche Bearbeitung“ [23]. Allein zwischen 1964 und 1972 entstanden in der städtischen Hautklinik 101 wissenschaftliche Publikationen.

Neben dem wissenschaftlichen Interesse besaß Weber einen ausgeprägten Erfindungsreichtum, gepaart mit dem nötigen Geschäftssinn, um eine Vielzahl von diagnostischen und therapeutischen Entwicklungen gemeinsam mit pharmazeutischen oder medizintechnischen Unternehmen umzusetzen, z.B. Volon A Schüttelmixtur (Heyden/Squibb, Regensburg) [25], Ligasano (Ligamed, Cadolzburg) [27] u.v.m.

Weitere Neuerungen unter Weber waren die Einrichtung eines Allergielabors, die Zuwendung zu Fragen der beruflichen Hautkrankheiten und die vermehrte Erstellung berufsdermatologischer Gutachten. Auch die erste in Nürnberg abgehaltene Tagung der Südwestdeutschen Dermatologenvereinigung 1966 wurde unter das Thema der Berufsdermatosen gestellt [23].

Im Jahr 1972 standen der Hautklinik 196 Betten (100 Männer, 91 Frauen, 5 Kinder) in Haus 19 zur Verfügung [23], durch die verbesserten Behandlungsmethoden, insbesondere durch die Einführung der Kortikosteroide („Von früh bis spat - Volonimat“) und neuerer systemischer Antibiotika, konnten die mittlere Verweildauer gesenkt und die Bettenzahl im weiteren Verlauf auf 157 Betten reduziert werden.

Im Oktober 1988 hatte Weber die Altersgrenze erreicht und schied aus der Klinik aus. Noch im gleichen Jahr eröffnete er eine dermatologische Praxis in der Kaiserstraße, 1991 wurde die PsoriSol-Hautklinik in Hersbruck eröffnet, deren medizinische Leitung er übernahm. Er blieb darüber hinaus auch wissenschaftlich aktiv und meldete mehrere Patente zum Thema Sonnenschutz an.

\section{Die Ära Eberhard Paul \\ $\nabla$}

Die Leitung der Hautklinik wurde zunächst kommissarisch vom leitenden Oberarzt Dr. Rolf Maier übernommen, bevor im Februar 1989 Prof. Dr. Eberhard Paul neuer Chefarzt wurde. Paul hatte in Gießen studiert, nach seiner Promotion am Anatomischen Institut wurde er Assistent an der Universitäts-Hautklinik bei Leonhard Illig (geb. 1920). 1980 wurde er habilitiert und 1986 Professor auf Lebenszeit. Gemeinsam mit Illig beschäftigte er sich mit der Urtikaria und dem Malignen Melanom [28]. Diese Themen blieben auch Schwerpunkte seiner Nürnberger Schaffenszeit.

Weitere wichtige Themenkomplexe waren das Diabetische Fußsyndrom und das venöse Ulcus cruris. Zur Erweiterung der Kenntnisse arterieller und venöser Gefäßerkrankungen hatte bereits Prof. Weber seinen Mitarbeiter Dr. Karl Heinz Galli an auswärtige Kliniken delegiert, insbesondere zu Prof. Dr. Wolfgang Hach an die William-Harvey-Klinik in Bad Nauheim. Galli, der zu Beginn seiner Medizinalassistentenzeit eine zweijährige chirurgische Ausbildung absolviert hatte, entwickelte in der Folge gemeinsam mit Paul ein Verfahren zur chirurgischen Sanierung des venösen Ulcus mit Dermatolipofasziosklerose, die sogenannte LAF-OP (Liposklerose-Abtragung, Fasziotomie), mit der hohe Heilungsraten erzielt werden konnten [29]. Die Liposkleroseabtragung gehört heute zum therapeutischen Standard, wurde jedoch unter dem Begriff "Shave-Therapie“ von anderen Autoren propagiert und unter diesem Begriff bekannt.

Für seine wissenschaftlichen Verdienste um die Melanomforschung wurde Paul im Jahr 2000 in das Royal College of Physicians and Surgeons in Glasgow aufgenommen und trägt seither den Titel FRCP (Glasg.). Für seine Verdienste um die ärztliche Fortbildung wurde ihm während der von ihm geleiteten Tagung

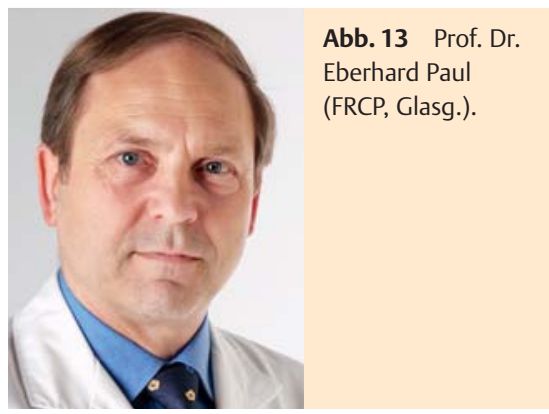

der Südostdeutschen Dermatologen 2002 die Ernst-von-Bergmann-Plakette der Bundesärztekammer verliehen. Paul war insbesondere zum Thema Vorbeugung und Früherkennung von Hautkrebs mit Vorträgen für die Bevölkerung unermüdlich um die Laienaufklärung bemüht, weitere Themen waren Allergien, chronische Wunden und das Ulcus cruris ( $\bullet$ Abb. 13).

Paul hatte 1989 die Nürnberger Hautklinik mit 120 Betten übernommen. Nach der Eröffnung des neu erbauten Klinikums Nürnberg Süd 1994 wurde die Verbrennungsstation in ein eigenes Zentrum für Schwerstbrandverletzte der Plastischen Chirurgie ausgelagert, die Hautklinik blieb mit 99 Betten eine der größten kommunalen Hautkliniken Deutschlands. Bei stetiger Absenkung der stationären Verweildauer stieg die Fallzahl kontinuierlich von 1989 bis 2001 um $60 \%$ an.

1998 wurde das Städtische Krankenhaus in ein selbstständiges gemeinnütziges Kommunalunternehmen unter städtischer Trägerschaft umgewandelt. Zeitgleich wurde eine kooperative Klinikleitung eingeführt, ein bundesweit einmaliges Führungskonzept [30]. Pflegedienstleitungen waren seither Ansbert Alex (1978-2000), Bernhard Szerbinski (2001 - 2008) und Hannelore Erb (seit 2008).

Im März 2002 wurde, nach Umwidmung und Sanierung einer früheren Bettenstation, eine Tagesklinik mit zunächst 17 Plätzen eröffnet, Indikationen waren insbesondere die Krankheitsbilder Psoriasis, Ekzeme und Ulcus cruris. Im stationären Bereich blieben zunächst 82 Betten erhalten, diese wurden bis 2007 schrittweise auf 67 Betten reduziert. Dies war nur möglich durch weitere Verkürzung der Verweildauer auf zuletzt 8 Tage.

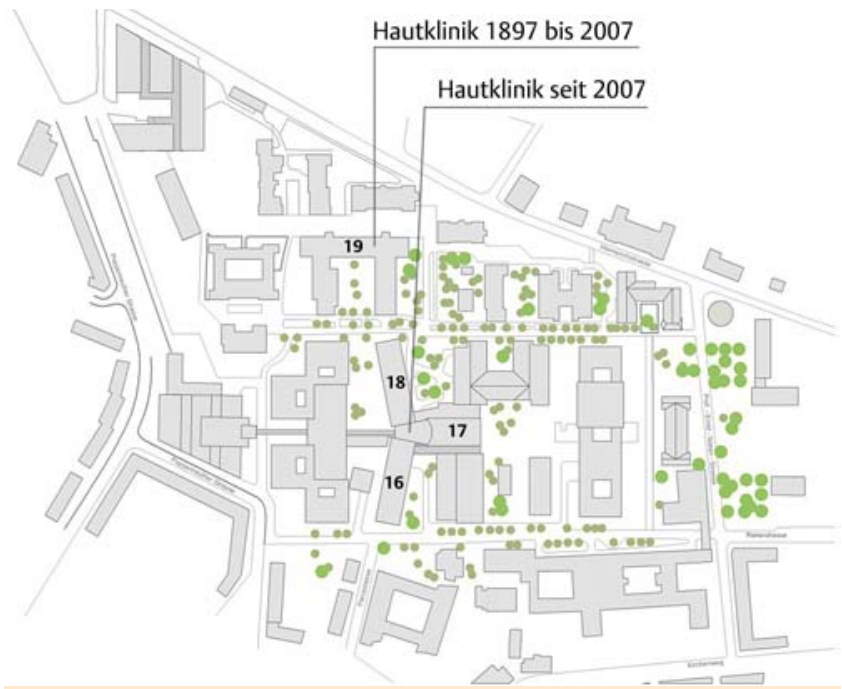

Abb. 14 Die aktuelle bauliche Situation des Klinikums Nürnberg Nord. Seit 1953/54 besteht der U-förmige „Bau 19“. Seit 2007 befindet sich die Hautklinik im sog. Y-Bau. Die Bauten 2 bis 7 sind der Baustelle für den Neubau Ost des „Zentrums für Altersmedizin“ gewichen. 


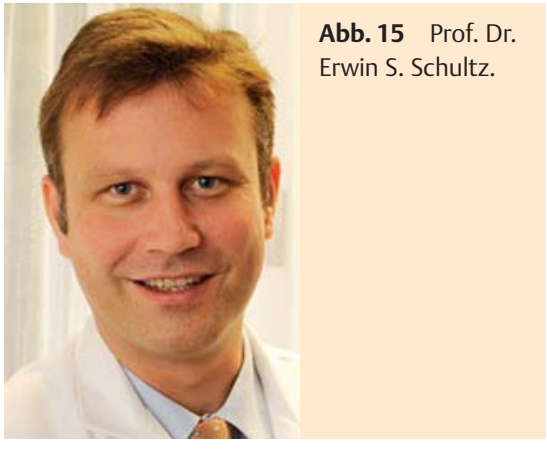

Im Jahre 2007 war die Ära des Bau 19 als Stammhaus der Hautund Geschlechtskrankheiten am Städtischen Krankenhaus seit 1897 zu Ende, die Hautklinik zog nach 110 Jahren in den sog. „YBau“ um: In der dritten und vierten Etage der Häuser 16, 17 und 18 konnten komplett neu renovierte Räumlichkeiten bezogen werden ( $\triangle$ Abb. 14).

Um den reibungslosen Umzug und Neustart zu gewährleisten, setzte Prof. Paul über die Altersgrenze hinaus noch für ein weiteres Jahr die Leitung der Hautklinik fort und ging mit Ablauf August 2008 in den „Ruhestand“. Seit Oktober 2008 betreibt er eine Privatpraxis für Dermatologie im Nürnberger Stadtteil Mögeldorf.

\section{Gegenwart und Ausblick \\ $\nabla$}

Im September 2008 wurde Prof. Dr. Erwin Schultz zum neuen Chefarzt der Hautklinik berufen ( Abb. 15). Nach dem Studium und der Promotion in Würzburg absolvierte er seine Facharztausbildung an der Dermatologischen Universitätsklinik in Erlangen unter Otto Paul Hornstein und Gerold Schuler. Nach einem Forschungsaufenthalt bei Pierre van der Bruggen am Ludwig Institute for Cancer Research in Brüssel habilitierte er sich zum Thema „Grundlagen der aktiven Immuntherapie des malignen Melanoms“ und war bis 2005 Oberarzt für den Bereich Dermatoonkologie. Es folgte der Ruf auf eine Professur für Dermatologie an der Philipps-Universität Marburg verbunden mit der Position des leitenden Oberarztes und stellvertretenden Klinikdirektors der Universitätshautklinik bei Michael Hertl. Auch in Nürnberg gilt sein Interesse insbesondere dem Ausbau des dermatoonkologischen Schwerpunktes.

So konnte innerhalb eines Jahres ein Hauttumorzentrum nach den Vorgaben der Deutschen Krebsgesellschaft etabliert und im Oktober 2009 zertifiziert werden. Damit bestand in Nürnberg das erste zertifizierte Hauttumorzentrum in Bayern. Um das bestehende dermatoonkologische Therapiespektrum zu erweitern, konnten zahlreiche internationale Therapiestudien initiiert werden.

Im Juli 2009 wurde, gemeinsam mit der Hals-, Nasen- und Ohrenklinik, der Kinderklinik und den Kliniken für Pneumologie und Gastroenterologie, das Nürnberger Interdisziplinäre Zentrum für Allergologie (NIZA) gegründet.

Heute wird in der Hautklinik am Klinikum Nürnberg auf höchstem Niveau die gesamte Palette der Diagnostik und Therapie dermatologischer Erkrankungen angeboten, insbesondere für Hauttumore und chronisch-entzündliche Hauterkrankungen sowie chronische Wunden. Von ihrem ursprünglichen Schwerpunkt - den Geschlechts- und Infektionskrankheiten - hat sich die Klinik deutlich entfernt, ohne diesen jedoch vollständig auf-

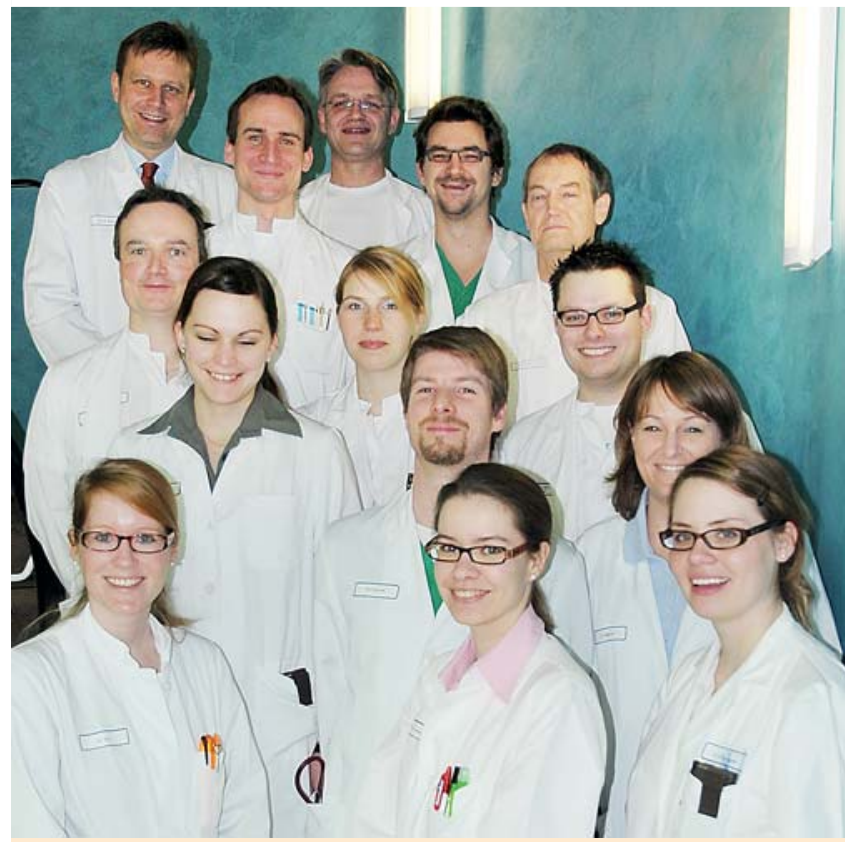

Abb. 16 Das ärztliche Kollegium im März 2010.

zugeben. Die Dermatologie stellt im Verbund mit den übrigen Kliniken des Klinikums Nürnberg als Haus der Maximalversorgung einen wichtigen Bestandteil dar und wird sich auch in $\mathrm{Zu}$ kunft als eigenständiges wie auch als interdisziplinär kooperierendes Fach behaupten. Es besteht Anlass zu der Annahme, dass die demografische Entwicklung, insbesondere mit der Zunahme an Hauttumoren und chronischen Wunden, dazu beitragen wird (॰ Abb. 16).

\section{Danksagung \\ $\nabla$}

Für die Hilfe bei der Quellensuche danke ich Herrn Jochem, Herrn Gebhardt und Frau Scholz vom Stadtarchiv Nürnberg.

Für die Überlassung von Krankenhaus-Festschriften und Fotografien danke ich Herrn Mossner, Herrn Funke und Herrn Bartl, Abteilung Bau und Technik, Klinikum Nürnberg.

Für persönliche Berichte zur Geschichte der Hautklinik danke ich Herrn Alex und Sr. Evelyne Peters-Sachs.

Für die Begeisterung an der Geschichte unseres Fachgebietes und für die klinische Ausbildung danke ich Herrn Dr. Christoph Löser, Herrn Dr. Karl Heinz Galli und Herrn Prof. Dr. Eberhard Paul.

\section{Abstract}

\section{Division Skin and Love - History of the Department for Dermatology and Venerology at Nuremberg Hospital $\nabla$}

In 2010, the Department of Dermatology and Venerology of Nuremberg Hospital can look back on a history of 165 years. Its precursor, the former Institute for Incurable Diseases, founded in 1813 at St. Sebastian Hospital, was replaced by the Department of Dermatology and Venerology in 1845, which was located in the newly built General Hospital at Sandstraße. Since 1987 the Department has been part of the New Communal Hospital in St. Johannis (today's Nuremberg North Hospital). In 2007, after 110 years, the Department moved to the renovated „Y-Building“. 
The following article will describe the history of the Department as far as important individuals, contemporary health care and some of the medical and structural conditions are concerned.

\section{Literatur}

1 Murken AH. Aus der Nürnberger Krankenhausgeschichte: Der bauliche Wandel des Allgemeinen Krankenhauses der Stadt Nürnberg im 19. Jahrhundert. Historia Hospitalium, Zeitschrift der Deutschen Gesellschaft für Krankenhausgeschichte 1974; 9

2 Lochner GF, Bock FL. Statistisch-medizinischer Bericht über die Kranken- und Versorgungsanstalten Nürnbergs. Nürnberg: Bauer und Raspe, 1844

3 Thoben C. Prostitution in Nürnberg: Wahrnehmung und Maßregelung zwischen 1871 und 1945. Neustadt a. d. Aisch: Schmidt, 2007

4 Geist L. Das allgemeine Krankenhaus der Stadt Nürnberg in den ersten zwanzig Jahren seines Bestehens, 1845/46 mit 1864/65. Nürnberg: Friedrich Korn'sche Buchhandlung, 1866

5 Windsheimer B. 100 Jahre Klinikum Nürnberg. Die Geschichte des Nürnberger Gesundheitswesens im späten 19. und 20. Jahrhundert. Nürnberg: Tümmels, 1997

6 Scholz A. Geschichte der Dermatologie in Deutschland. Heidelberg: Springer, 1999

7 Städtische Kollegien (Hrsg). Das Allgemeine Krankenhaus der Stadt Nürnberg. Festschrift zur Eröffnung des Neuen Krankenhauses der Stadt Nürnberg. Nürnberg: Selbstverlag des Magistrats, 1898

8 Plank R. Gesundheitswesen. In: Luppe H (Hrsg). Jahresschau Nürnberg 1923/24. Nürnberg: Montanus, 1924

9 Löser C, Plewig G. Pantheon der Dermatologie. Herausragende historische Persönlichkeiten. Heidelberg: Springer, 2008

10 Riecke E. Deutscher Dermatologenkalender. Leipzig: Barth, 1929

11 Rothmann HL. Geschlechtskrankheiten und Prostitution in Nürnberg 1870 - 1930. Eine medizinhistorische Quellenstudie [Dissertation]. Erlangen: Universität Erlangen, 1952

12 Streicher J. Verjudung des Krankenhauses. Der Stürmer, Nürnberger Wochenblatt zum Kampf um die Wahrheit. Oktober 1923, Nr. 16: 3

13 Vasold M. Das Städtische Krankenhaus Nürnberg während des Dritten Reiches. Zeitschrift für Bayerische Landesgeschichte 1998; 61: 763 826
14 Landes E. Charakterköpfe der Frankfurter Medizinischen Fakultät. Ein Beitrag zur Dermatologie in Frankfurt am Main. Hessisches Ärzteblatt 1991; 10: 516

15 Riecke E. Deutsches Dermatologen-Verzeichnis. Leipzig: Barth, 1939

16 Fischer I, Voswinkel P. Biographisches Lexikon der hervorragenden Ärzte der letzten 50 Jahre. Nachträge und Ergänzungen. Hildesheim: Olms, 2002

17 Scholz A, Holubar K, Burg G. Geschichte der Deutschsprachigen Dermatologie. Weinheim: Wiley-VCH, 2009

18 Anonym. Friedrich-Wilhelm Dietel. 75 Jahre am 2. September 1972. Dermatologische Monatsschrift 1972; 158: 692

19 Wittern R. Die Professoren und Dozenten der Friedrich-Alexander-Universität Erlangen 1743 -1960. Erlangen: Friedrich-Alexander-Universität Erlangen-Nürnberg, 1999

20 Funk CF, Walther H. Friedrich W. Dietel zum 60. Geburtstag am 2. September 1957. Dermatologische Monatsschrift 1957; 37 (136): 981 982

21 Stadtrat Nürnberg (Hrsg). „50 Jahre Allgemeines Städtisches Krankenhaus Nürnberg“, Denkschrift anlässlich des 50jährigen Bestehens des Allgemeinen Städtischen Krankenhauses Nürnberg. Nürnberg: Tümmels, 1948

22 Mitteilung von Herrn Ansbert Alex, von 1956 bis 2000 Krankenpfleger der Hautklinik, ab 1978 als Pflegedienstleitung.

2375 Jahre Städtische Krankenanstalten Nürnberg. Bericht für die Jahre 1947 - 1972. Nürnberg: 1972

24 Löhe H, Langer E. Die Dermatologen deutscher Sprache. Leipzig: Barth, 1955

25 Mitteilung von Sr. Evelyne Peters-Sachs, seit 1968 Krankenschwester in der Hautklinik, zuletzt pflegerische Bereichsleitung des OP.

26 Jung EG (Hrsg). 30 Jahre Aktuelle Dermatologie: ein Jubiläumsband. Stuttgart: Thieme, 2005

27 Weber G, Galli KH. Therapeutische Anwendung von Polyesterschaumstoff. Deutsches Ärzteblatt 1980; 25: 1621 - 1625

28 Paul E. Die Entwicklung der Hautklinik am Klinikum Nürnberg von 1989 bis 2006. Nürnberg: Selbstverlag, 2007

29 Galli KH, Wolf $H$, Paul E. Therapie des Ulcus cruris venosum unter Berücksichtigung neuerer pathogenetischer Gesichtspunkte. Phlebologie 1992; $21: 183-187$

30 Maly U, Estelmann A (Hrsg). Kommunal und erfolgreich. Das Klinikum Nürnberg im Spannungsfeld zwischen Wettbewerb und öffentlicher Daseinsfürsorge. Frankfurt: Mabuse, 2007 